\title{
HIV risk perception and sexual behaviors among female sex workers in Tehran, Iran
}

\author{
Zahra Jorjoran Shushtari ${ }^{1,2}$, Seyed Ali Hosseini*1, Homeira Sajjadi ${ }^{3}$, Yahya Salimi ${ }^{4}$, Armita Shahesmaeili $^{5}$, \\ Tom A.B. Snijders ${ }^{6,7}$
}

\section{Abstract}

Background: An adequate perception of the degree to which one is at risk of having or contracting HIV is necessary for behavioural change and the adoption of safe behaviours. There are limited data regarding HIV risk perceptions among female sex workers in Iran. This study aimed to determine the HIV risk perception status and its association with sexual behaviours among female sex workers in Tehran.

Methods: A cross sectional study was conducted among 170 female sex workers in Tehran. Participants were recruited using a combination of snowball, purposeful, and convenience sampling methods. Multiple logistic regression was used to identify adjusted associations between background factors, sexual behaviours, and HIV risk perception. The analysis was conducted by the "logistf" package in the R statistical system. P-value less than .05 was considered as statistically significant.

Results: Among the participants, 122 (77\%) reported high HIV risk perception. Most female sex workers with high HIV risk perception reported that they did not consistently use condoms $(n=120,98 \%$. Female sex workers with a higher frequency of sex work ( $\mathrm{AOR}=1.18,95 \%$ CI: 1.08, 1.31), inconsistent condom use (AOR $=0.15,95 \%$ CI: 0.02, 0.66), a history of $\mathrm{HIV}$ testing $(\mathrm{AOR}=5.1,95 \%$ CI: $1.2,26.0)$, and low HIV knowledge ( $\mathrm{AOR}=0.97,95 \% \mathrm{CI}=0.95,0.996)$ were more likely to report high HIV risk perception.

Conclusion: Most female sex workers with risky sexual behaviours had a high HIV risk perception. Effective educational programs are suggested to enable female sex workers to correctly assess their own HIV risk and change risk behaviors based on self-assessment of actual risk.

Keywords: HIV risk perception, Sexual behaviours, HIV/AIDS, Female sex workers

Conflicts of Interest: None declared

Funding: None

*This work has been published under CC BY-NC-SA 1.0 license.

Copyright $\odot$ Iran University of Medical Sciences

Cite this article as: Jorjoran Shushtari Z, Hosseini SA, Sajjadi H, Salimi Y, Shahesmaeili A, Snijders TAB. HIV risk perception and sexual behaviors among female sex workers in Tehran, Iran. Med J Islam Repub Iran. 2019 (25 Sep);33:101. https://doi.org/10.47176/mjiri.33.101

\section{Introduction}

Globally, female sex workers (FSWs) are among the most vulnerable groups who are disproportionately affected by the HIV/AIDS pandemic (1-3). A review study about HIV burden in female sex workers in 50 low-and

\footnotetext{
Corresponding author: Dr Seyed Ali Hosseini, al.hoseini@uswr.ac.ir
}

1. Social Determinants of Health Research Center, University of Social Welfare and Rehabilitation Sciences, Tehran, Iran

2. Student Research Committee, University of Social Welfare and Rehabilitation Sciences, Tehran, Iran

3. Social Welfare Management Research Center, University of Social Welfare and Rehabilitation Sciences, Tehran, Iran

4. Social Development and Health Promotion Research Center, Health institute, Kermanshah University of Medical Sciences, Kermanshah, Iran

5. HIV/STI Surveillance Research Center, and WHO Collaborating Center for HIV Surveillance, Institute for Futures Studies in Health, Kerman University of Medical Sciences, Kerman, Iran

6. Department of Sociology, University of Groningen, 9712 TG Groningen, Netherlands

7. Nuffield College, University of Oxford, OX1 1NF Oxford, United Kingdom middle-income countries reported $11.8 \%$ HIV prevalence, with the pooled odds of HIV infection being 13.5 times higher for them than for the general population of women in the reproductive age group (1). Recently, the main pat-

$\uparrow$ What is "already known" in this topic:

An adequate perception of the degree to which one is at risk of having or contracting HIV is necessary for behavioural change and adoption of safe behaviours.

$\rightarrow$ What this article adds:

Female sex workers with risky sexual behaviours had high HIV risk perception. However, there seem to be misperceptions in this population regarding their own HIV risk. Many female sex workers with high HIV knowledge perceived themselves to be at low HIV risk. Thus, HIV education programs need to be enhanced to translate female sex workers' knowledge of HIV into accurate risk perceptions. 
tern of HIV transmission in many countries, including Iran, has shifted from injecting drug use to sexual transmission (4-6).

Female sex workers are a hidden and hard to reach population, and they can play a key role in the HIV epidemic because of their risk of sexual and drug use behaviours. The number of FSWs in Iran has been estimated as 228 $700(95 \%$ CI 153,500, 294,300) and the HIV prevalence among them was estimated $4.5 \%$ in $2015(6,7)$. Also, the number of FSWs in Tehran was estimated as $38700(95 \%$ CI, 34 200, 43 400) (7).

In Iran, Ministry of Health and Medical Education have done enormous investments and preventive efforts to increase the access of HIV at-risk population (FSWs) to HIV prevention programs such as drop-in centres (DIC), shelters, consultation behavioural centres, and especially vulnerable women's consultation centres throughout the country. These centres provide free services, including basic sexual and reproductive health care, educational programs about sexual transmitted infections (STI) and prevention methods, HIV testing, and counselling $(8,9)$. Based on recent evidence, it seems that prevalence of HIV risk behaviours is still relatively high among FSWs (10, 11). Some studies reported that many FSWs had some information about HIV/STI transmission and prevention methods; however, this information was not always used in practice $(10,12)$. One study among FSWs in Shiraz, a city in South of Iran, reported that most participants (95\%) knew that condoms can prevent HIV, but only $36 \%$ used condoms with their clients and $25 \%$ with their non-paying partners (10). An idea is that FSWs may underestimate their risks and vulnerabilities, specifically for getting HIV. This underestimation sometimes might be compounded by mental health problems such as cognitive dissonance, as FSWs may perceive that measures to reduce these risks would endanger their livelihood. Vulnerable and marginalized groups may not have enough control over their lives, and they may even feel the need to do risky things for survival. Thus, they justify their behaviour by a low HIV risk perception.

Risk and vulnerability perception is a complex and multifaceted concept that depends on behaviours and experiences of people during their life course, and it is directly or indirectly influenced by socioeconomic status, political, and cultural factors $(13,14)$. According to the literature on health-related behaviour, people's awareness and perception of their degree of being at risk of infections such as HIV is necessary for behavioural changes and adopting preventive behaviours (15-17). Various models and theories have been proposed regarding the mechanisms that may be linked to risk perception, such as behaviours and skills (18), health beliefs, social learning (19), reasoned actions, and planned behaviour (19). Psychosocial/cognitive models of HIV prevention suggest that 'incorrect' risk perceptions, which may go as far as a sense of invulnerability, constitute a major barrier to protective and preventive behaviours such as condom use and HIV testing (20). A population-based study among adolescent and young adults in Mozambique indicated that HIV risk perception was positively associated with condom use
(21). A study among men who have sex with men (MSM) showed that perceived HIV risk is an important predictor for low adoption of risky behaviours (22). A longitudinal study among youths in South Africa showed that change toward high risk perception was significantly associated with change to more HIV testing behaviours (23). Several studies assessing risk perception found that HIV knowledge has effects on risk perception and on taking risky behaviours $(14,24,25)$. Some people are aware of the risk of getting HIV through sexual contact, but they may think this risk is only associated with a work setting in which people do not know their partners, and not with sexual contacts with their regular partner $(14,24)$.

Accurate self-assessment of HIV risk perception can provide valid data to identify how people think and feel about health outcomes related to sexual risk activity (26, 27). Availability of data that are locally relevant can help to improve understanding of FSWs' HIV risk perception and its effect on their sexual behaviours. Such information is also critical for guiding policy and developing effective prevention programs. Programs focusing on FSWs with high risk perception can be an important strategy in a resource-limited setting, such as Iran, to achieve maximum benefits from preventive interventions among FSWs. Although there are some studies about HIV risk perception and risk behaviours among different populations, such as injecting drug users $(28,29)$, MSM (22), and HIV positive people, there is limited information on HIV risk perception and its association with sexual behaviours among FSWs, especially in Iran. This study aimed to determine perceptions of HIV risk perception and their association with sexual behaviours among FSWs in Iran.

\section{Methods}

\section{Study sample and procedure}

This cross sectional study was conducted among 170 FSWs in Tehran from February 2016 to December 2017. First, explorative field work was done to identify the sites of working and living of FSWs. A combination of snowball, purposeful, and convenience sampling methods that have been successfully used in previous studies to recruit 'hidden' populations $(30,31)$ was used to recruit FSWs from all involved sites, which included drop-in centers (DICs), shelters, voluntary consulting centers, and outreach spots. Eligibility criteria were being 16 years or older, having had sex for money in the last year, identifying oneself as sex worker, and willingness to participate in the study. Sex work was operationalized as engaging in sexual relations to exchange for something else such as money, drugs, gifts, and shelter. For the snowball sampling, to initiate the chain referral process, 6 seed participants who satisfied the inclusion criteria were selected using convenience sampling. Each recruited participant was asked to introduce other FSWs who satisfied the inclusion criteria, which then constituted the next wave, and this process continued for 5 waves. Each participant received an incentive (100 000 Rails equal to 3 USD) to compensate for their time. They also received a secondary incentive (same amount) if they recruited other eligible FSWs. Many interviews were conducted individually in a private room in 
DICs. However, for some FSWs who were unwilling to participate in the interviews in DICs, interviews were completed at a park or their home. To improve diversity from all involved sites, purposeful sampling from outreach spots, such as group homes, streets, and parks, was done by a peer outreach worker who collaborated with the study via drop-in centers. Convenience sampling was used to recruit the participants among FSWs who attended DICs. No written informed consent was obtained from the participants, as it would have endangered the anonymity of the FSWs; sex work is illegal in Iran. Therefore, verbal informed consent was received for all interviews.

All participants were provided with explanations about the purpose of the study, and they were informed about the confidentiality of their information and their right to withdraw from the study at any time. The study protocol was reviewed and approved by the Ethics Committee of Tehran University of Social Welfare and Rehabilitation Sciences (IR.USWR.REC.1394.187).

\section{Measures}

To assess HIV risk perception, the participants were asked the following question:

"How much do you think you are at HIV risk?" The answers were rated based on a 5-point Likert response $(1=$ very low, $2=$ low, $3=$ somewhat, $4=$ much, and $5=$ very much). This Likert response was changed to a binary code, including high HIV risk perception $(=1)$ and low HIV risk perception $(=0)$ for the data analysis. Previous studies reported acceptable reliability and validity for this single item to measure HIV risk perception $(32,33)$.

Sexual behaviors were assessed based on several variables, including age of initiating sex work, frequency of sex work in the last month, unplanned sex- interpreted as risky sexual behaviors- and having a HIV test, and condom useinterpreted as protective sexual behaviors. The age of initiating sex work was measured as a categorical variable with the following categories: before 18, between 18-30 years, and after 30 years. The frequency of sex work in the last month was recorded as a count variable. For this analysis, condom use in the last month was dichotomized into consistent condom use and inconsistent condom use. HIV Knowledge was measured by a 14 -item questionnaire that was established in Iranian population (34). In this study, Cronbach's alpha for this scale was 0.86 . The total score of HIV knowledge transformed to a scale of 0 to 100 , with high scores indicating more knowledge about HIV. To measure HIV testing status, the participants were asked if they have ever had a HIV test? With a binary response (yes or no). Participants were asked to recall any unplanned sex in the last month with a binary response (yes or no). The present study included questions about sociodemographic characteristics including age, monthly income, and place of living. Age and education were considered as continuous variables. Place of living was categorized into 3 groups: homeless, living in other's home, and living in their own home.

\section{Statistical analysis}

The distribution of the scores were assessed carefully for missing values and outliers. Frequency of sex work had 3 values larger than 20 (two values 25 , one 60 ), which were considered outliers and were truncated to 20 .

Low and high HIV risk perception subgroups were compared based on the demographics, sexual behaviors, and experiences of the participants using Chi-square and $t$ test.

The adjusted associations between HIV risk perception and sexual behaviors were estimated using the multiple logistic regression model. The linearity assumption was checked for continuous variables. The variables that were included in the multiple logistic regression model were selected based on the literature review and available evidence $(15,22,35-37)$. Using a conceptual framework, it was expected that HIV risk perception, as the dependent variable, would have a positive association with protective sexual behaviors, HIV testing and condom use, and a negative association with risky sexual behaviors, age of initiating sex work, frequency of sex work in the last month, and unplanned sex. The independent variables were entered into the multiple logistic regression model in 2 models. The first model included sociodemographic characteristics (age, education, and place of living), risky sexual behaviors (age of initiating sex work, number of sex work in the last month, and unplanned sex in the last month), and HIV knowledge. The second model included these variables and also protective sexual behaviors variables (history of HIV testing and consistent condom use). The reason for this distinction between the 2 models is that HIV testing, condom use, and HIV risk perception could affect one another causally in any direction, while for the independent variables in the first model it may be more acceptable to assume that they are causal antecedents to risk perception. However, this assumption was not firmly made.

Because several combinations of the independent variables occurred very infrequently in the data, to avoid sparse data bias, parameters were estimated by penalized likelihood according to Firth's method (38-40). The adjusted odds ratio (AOR), its 95\% confidence interval, and pvalue were calculated using profile likelihood (41). The analysis was conducted by the "logistf" package in the $\mathrm{R}$ statistical system.

\section{Results}

The total number of the participants was 170 , of whom $11(6 \%)$ were HIV positive who knew their status. The data related to these HIV positive participants who knew their HIV status were excluded from the study. The data for participants who were HIV negative or had unknown HIV status were used for the analysis in this study $(\mathrm{N}=159)$. The sociodemographic and sexual behaviors characteristics of the participants are presented in detail in Table 1.

The mean age of the participants was $34.4(\mathrm{SD}=7.6)$. Among the participants, 42 (26\%) were homeless, 50 (31\%) reported living at other's home, and 67 (42\%) lived in their personal home. The mean score of HIV knowledge of the FSWs was 77.6 (19.6). Of 159 FSWs who participated in this study, $122(77 \%)$ reported high 
Table 1. Characteristics of the study sample by HIV risk perception in the participants of the study

\begin{tabular}{|c|c|c|c|c|}
\hline \multirow[t]{3}{*}{ Characteristics } & & \multicolumn{2}{|c|}{ HIV risk perception } & \multirow[t]{3}{*}{$\mathrm{p}$} \\
\hline & & $\operatorname{High}(\mathrm{n}=122)$ & Low $(n=37)$ & \\
\hline & & $\mathrm{N}(\%)$ or Mean $(\mathrm{SD})$ & $\mathrm{N}(\%)$ or Mean $(\mathrm{SD})$ & \\
\hline Age & & $34.0(\mathrm{SD}=7.3)$ & $35.5(\mathrm{SD}=8.3)$ & 0.337 \\
\hline \multirow{2}{*}{ Marital status } & Single & $25(20 \%)$ & $7(19 \%)$ & 1.000 \\
\hline & Married & $97(80 \%)$ & $30(81 \%)$ & \\
\hline \multirow{6}{*}{ Educational level } & Illiterate & $5(4 \%)$ & $0(0 \%)$ & 0.005 \\
\hline & Just reading and writing & $2(2 \%)$ & $0(0 \%)$ & \\
\hline & Primary education & $14(13 \%)$ & $2(5 \%)$ & \\
\hline & secondary education & $52(43 \%)$ & $11(30 \%)$ & \\
\hline & High school or Diploma & $48(39 \%)$ & $20(54 \%)$ & \\
\hline & University degree & $1(1 \%)$ & $4(11 \%)$ & \\
\hline \multirow[t]{3}{*}{ Place of living } & Homeless & $35(29 \%)$ & $7(19 \%)$ & 0.434 \\
\hline & Living in others' home & $36(30 \%)$ & $14(38 \%)$ & \\
\hline & Personal home & $51(42 \%)$ & $16(43 \%)$ & \\
\hline \multirow[t]{3}{*}{ Age of initiating sex work } & $<18$ & $16(13 \%)$ & $2(5 \%)$ & 0.570 \\
\hline & $18-30$ & $90(74 \%)$ & $26(70 \%)$ & \\
\hline & $\geq 30$ & $16(13 \%)$ & $9(24 \%)$ & \\
\hline \multicolumn{2}{|l|}{ Frequency of sex work in the last month } & $11.0(\mathrm{SD}=4.9)$ & $8.0(\mathrm{SD}=9.6)$ & $<0.001$ \\
\hline \multirow[t]{2}{*}{ Unplanned sex } & Yes & $93(76 \%)$ & $20(54 \%)$ & 0.016 \\
\hline & No & $29(24 \%)$ & $17(46 \%)$ & \\
\hline \multirow[t]{2}{*}{ Consistent condom use in the last month } & Yes & $2(2 \%)$ & $10(27 \%)$ & \\
\hline & No & $120(98 \%)$ & $27(73 \%)$ & \\
\hline \multirow{2}{*}{ History of HIV testing } & Yes & $102(84 \%)$ & $26(70 \%)$ & 0.119 \\
\hline & No & $20(16 \%)$ & $11(30 \%)$ & \\
\hline HIV knowledge & & $75.4(\mathrm{SD}=20.8)$ & $85.1(\mathrm{SD}=12.7)$ & $<0.001$ \\
\hline
\end{tabular}

HIV risk perception. Many of the FSWs with high risk perception reported secondary education $(n=52,43 \%)$. However, most of the FSWs with low risk perception had high school education level or high school diploma $(n=20$, $54 \%$ ). The FSWs with high risk perception compared to low risk perception were more likely to have high frequencies of sex work (Mean $11.0 \quad(\mathrm{SD}=4.9)$ vs 8.0 $(\mathrm{SD}=9.6), \mathrm{p}<0.001)$, unplanned $\operatorname{sex}(76 \%$ vs $54 \%$, $\mathrm{p}=0.0160)$, and history of HIV testing $(84 \%$ vs $70 \%$, $\mathrm{p}=0.119)$. Most FSWs with high HIV risk perception reported that they did not consistently use condoms (98\%). Table 1 shows the HIV risk perception status among different subgroups of the participants.
The multiple logistic regression results are presented in Table 2. The 2 models agree with respect to the significant effects of all variables included in both models. Results for the first model are as follow: One level increase in education decreased the odds of high risk perception by $59 \%(\mathrm{AOR}=0.41,95 \% \mathrm{CI}=0.20,0.73)$. A higher frequency of sex work significantly increased the odds of reporting high-risk perception by $18 \%(\mathrm{AOR}=1.18,95 \% \mathrm{CI}$ : $1.08,1.31)$. One-point increase in HIV knowledge score significantly decreased the odds of high risk perception by $3 \%(\mathrm{AOR}=0.97,95 \% \mathrm{CI}=0.95,0.996)$, which may seem small, but the standard deviation of HIV knowledge was 19.6, thus one standard deviation increase in HIV

Table 2. Adjusted association between HIV risk perception and sexual behaviors of the FSWs in the multiple logistic regression analysis

\begin{tabular}{|c|c|c|c|c|c|}
\hline \multirow[b]{3}{*}{ Independent variables } & & \multicolumn{4}{|c|}{ Dependent variable (HIV risk perception) } \\
\hline & & \multicolumn{2}{|c|}{ Model 1} & \multicolumn{2}{|c|}{ Model 2} \\
\hline & & AOR & (CI 95\%) & AOR & $(\mathrm{CI} 95 \%)$ \\
\hline$\overline{\text { Age }}$ & & 0.99 & $0.91,1.06$ & 1.01 & $0.93,1.10$ \\
\hline Education & & 0.41 & $0.20,0.73 *$ & 0.51 & $0.24,0.96^{*}$ \\
\hline \multirow[t]{3}{*}{ Place of living } & Homeless & - & - & - & - \\
\hline & Living in others' home & 0.50 & $0.15,1.56$ & 0.80 & $0.22,2.73$ \\
\hline & Personal home & 0.82 & $0.30,2.46$ & 0.94 & $0.27,3.07$ \\
\hline \multirow[t]{3}{*}{ Age of initiating sex work } & $<18$ & - & - & - & - \\
\hline & $18-30$ & 1.26 & $0.20,5.84$ & 1.05 & $0.15,5.43$ \\
\hline & $\geq 30$ & 0.93 & $0.10,7.5$ & 0.79 & $0.07,7.72$ \\
\hline Frequency of sex work in the last month & & 1.18 & $1.08,1.31^{*}$ & 1.131 .1 & $1.02,1.25^{*}$ \\
\hline \multirow[t]{2}{*}{ Unplanned sex } & No & 1.46 & $0.60,3.50$ & 1.39 & $0.53,3.54$ \\
\hline & Yes & - & - & - & - \\
\hline \multicolumn{2}{|l|}{ HIV knowledge } & 0.97 & $0.95,0.996^{*}$ & 0.96 & $0.92,0.99 *$ \\
\hline \multirow[t]{2}{*}{ Consistent condom use in the last month } & Yes & & & 0.15 & $0.02,0.66^{*}$ \\
\hline & No & - & - & - & - \\
\hline \multirow{2}{*}{ History of HIV testing } & No & - & - & - & - \\
\hline & Yes & & & 5.08 & $1.23,26.0 *$ \\
\hline
\end{tabular}


knowledge decreased the odds of high risk perception by $42 \%(\mathrm{AOR}=0.58, \mathrm{CI}=0.33,0.92)$. Having unplanned sex did not significantly affect risk perception. The results for the 2 variables added for Model 2 are as follow: Consistent condom use in the last month significantly decreased the odds of high risk perception by $86 \%$ (AOR $=0.15,95 \% \mathrm{CI}: 0.02,0.66)$. FSWs who have had a HIV test were much more likely to report high risk perception compared to those who did not have HIV test (AOR=5.1, 95\% CI: 1.2, 26.0).

\section{Discussion}

The present study adds to the growing body of literature about HIV risk perception and sexual behaviors that may help and contribute to designing HIV prevention interventions among FSWs. In this study, a sample of 159 FSWs was examined in Tehran, Iran. Most of these FSWs $(\mathrm{N}=122,77 \%)$ perceived themselves to be at high risk for HIV. This finding is consistent with evidence showing that FSWs in Iran are at heightened risk for HIV infection. Iran is in the third phase of HIV prevalence in which sexual transmission is steadily growing $(6,42)$. Results of this study showed that participants who had a higher frequency of sex work in the last month were more likely to perceive themselves to be at high risk for HIV. Several studies found that having multiple sexual partners, which may lead to a higher number of sexual relationships, is associated with an increased perceived risk for HIV infection $(15,22,29)$. The findings of this study also indicated that FSWs with high HIV knowledge perceived themselves at lower risk for HIV infection. This is consistent with some previous studies $(22,36,43)$, but inconsistent with others $(16,44)$. Indeed, one can argue both ways with respect to how HIV knowledge might translate into the assessment of one's own level of risk. A person may think she is at low risk of HIV just because she has more HIV knowledge. Also, a person, despite having a good general knowledge about HIV, may deny the possibility of having such a disease because she harbors incorrect assumptions about trust in sexual relationships and monogamy of her sexual partner, and also perceives no symptoms of sexually transmission infections. Another possibility is that FSWs who have more HIV knowledge may use condoms more and, therefore, perceive themselves to be at low-risk for HIV. The inconsistency in the results of different studies can be understood from these complexities in the association between HIV risk perception and HIV knowledge. This association may further depend on different cultures and many social factors, which should be considered in future studies. Having knowledge about HIV transmission without acknowledging risky behaviors of oneself and one's sexual partners, may be insufficient to motivate behavior change (45). Therefore, HIV education programs need to be enhanced to translate FSWs' knowledge of HIV into accurate risk perceptions.

One of the interesting findings was that the FSWs who had HIV testing were more likely to perceive themselves to be at risk for HIV than those who had not tested for HIV. One possible explanation for this finding is that FSWs who engage in risk behaviors may feel to be at high risk for HIV and seek more HIV test services. Therefore, low risk perception among FSWs can be a barrier for HIV testing. Our finding suggests that it is necessary to provide educational programs to enable FSWs to correctly judge their own risk for getting HIV and to encourage them for HIV testing. On the other hand, improving accessibility of HIV testing for FSWs can be an important strategy for HIV prevention among this group. This finding is consistent with results of previous studies among FSWs and rural women $(36,46)$. However, there is a controversy in the literature about the association of risk perception and HIV testing in different populations such as MSM, IDUs, and people with HIV. Some studies showed that people with high risk perception were more likely to have not tested for HIV (35), whereas some other studies showed that people who perceived themselves as having high HIV risk were more likely to have tested for HIV $(43,47)$. However, one study among MSM found no association between risk perception and HIV testing (22). This controversy in the literature may be due to differences in the studied populations, methodological aspects, and social and cultural contexts among the studies.

In line with the literature $(15,22,29)$, findings of this study indicate that the FSWs who inconsistently used condoms were more likely to perceive themselves to be at high risk for HIV compared to those FSWs who consistently used condoms. This finding may be due to various reasons such as absence of self-efficacy among FSWs, social norms unsupportive of condom use, and gender power inequality in sexual relationships of FSWs with their sexual partners. Therefore, it seems that these FSWs think they have to accept unsafe sex and accept the idea of being at risk. They need to get enough money to live on, so they do not have enough power to negotiate about safe sex behaviors with sexual partners because of fear of rejection by their sexual partners and fear of losing their emotional or financial supports $(48,49)$.

Findings of this study had 3 main limitations. First, no causal inferences were drawn due to the cross sectional nature of the study. Second, the results of this study might have been affected by measurement error, self-report data were used. Finally, the study participants were recruited using a nonrandom snowball sampling from one city in Iran. Therefore, the findings cannot be generalized to all FSWs in the country. In spite of these limitations, this study provided important insights about HIV risk perception and its association with sexual behaviors and experiences among FSWs that can be used for HIV intervention programs.

\section{Conclusion}

FSWs with risky sexual behaviors correctly perceived themselves to be at high risk of HIV. Therefore, it seems that they were aware of their individual risk behaviors. However, the finding that FSWs with more HIV knowledge considered themselves to be at lower risk, suggests that this population has misperceptions about the issue. Thus, educational programs are necessary to enable FSWs to correctly assess their own HIV risk and encourage behavioral change based on self-assessment of actual 
risk. Also, strategies should be targeted to change sexual practices among those who perceived to be at high risk.

\section{Acknowledgments}

This paper is part of a $\mathrm{PhD}$ thesis from University of Social Welfare and Rehabilitation Sciences, Tehran, Iran. All the study participants are appreciated for their contribution.

\section{Ethical approval}

Ethics approval for this study was obtained from the ethical review board of the University of Social Welfare and Rehabilitation Sciences.

\section{Conflict of Interests}

The authors declare that they have no competing interests.

\section{References}

1. Baral S, Beyrer C, Muessig K, Poteat T, Wirtz AL, Decker MR, et al. Burden of HIV among female sex workers in low-income and middle-income countries: a systematic review and meta-analysis. Lancet Infect Dis. 2012;12(7):538-49.

2. UNAIDS. Global AIDS Update 2016,. http://www.unaids.org/sites/ default/files/media_asset/global-AIDS-update-2016_en.pdf; 2016.

3. Organization WH. WHO Guidelines: Prevention and treatment of HIV and other sexually transmitted infections for sex workers in lowand middle-income countries. Geneva: World Health Organization. 2012.

4. Strathdee SA, Sherman SG. The role of sexual transmission of HIV infection among injection and non-injection drug users. J Urban Health. 2003;80(3):iii7-iii14.

5. Kral AH, Bluthenthal RN, Lorvick J, Gee L, Bacchetti P, Edlin BR. Sexual transmission of HIV-1 among injection drug users in San Francisco, USA: risk-factor analysis. Lancet. 2001;357(9266):1397401.

6. National AIDS Committee Secretariat, Ministry of Health and Medical Education. Islamic Republic of Iran AIDS Progress Report: On Monitoring of the United Nations General Assembly Special Session on HIV and AIDS. Tehran, Iran; 2015.

7. Sharifi H, Karamouzian M, Baneshi MR, Shokoohi M, Haghdoost A, McFarland W, et al. Population size estimation of female sex workers in Iran: Synthesis of methods and results. PLoS One. 2017;12(8): e0182755.

8. Education MoHaM, health Vco. protocol of vulnerable women's consultation centres Tehran, Iran. 2010

9. Establishment of a Drug Addiction Reducing Service Center, Drop in centers https://goums.ac.ir/files/deputy_health/c15.docx2006

10. Kazerooni PA, Sayadi M, Motazedian N, Sabet M. Sexual Behaviors, Knowledge and Attitudes of Female Sex Workers' towards HIV/AIDS in Shiraz. JHSSS. 2014;2(3):99-106.

11. Danial Z, Moayed M, Motamedi K, Hossain M, Mirhashemi S, Zamanian H. New Wave of Sexually-Transmitted HIV in Iran. HPR. 2017;2(1):25-6.

12. Kolahi AA, Rastegarpour A, Abadi AR, Nabavi M, Sayyarifard A, Sohrabi MR. The knowledge and attitudes of a female at-risk population towards the prevention of AIDS and sexually transmitted infections in Tehran. Journal of Research in Medical Sciences: J Res Med Sci. 2011;16(11):1452.

13. Taylor-Gooby P, Zinn JO. Current directions in risk research: new developments in psychology and sociology. Risk Anal. 2006;26(2):397-411.

14. Auli NC, Mejía-Lancheros C, Berenguera A, Mayans MV, Lasagabaster MA, Pujol-Ribera E. Risk perception of sexually transmitted infections and HIV in Nigerian commercial sex workers living in Barcelona: a study protocol. BMJ Open. 2013;3(7):e003345.

15. Shiferaw Y, Alemu A, Assefa A, Tesfaye B, Gibermedhin E, Amare M. Perception of risk of HIV and sexual risk behaviors among University students: implication for planning interventions. BMC Res Notes. 2014;7(1):162.
16. Bernardi L. Determinants of individual AIDS risk perception: knowledge, behavioural control and social influence. Afr J AIDS Res. 2002;1(2):111-24.

17. Darbes L, Crepaz N, Lyles C, Kennedy G, Rutherford G. The efficacy of behavioral interventions in reducing HIV risk behaviors and incident sexually transmitted diseases in heterosexual African Americans. AIDS. 2008;22(10):1177.

18. Fisher JD, Fisher WA. Changing AIDS-risk behavior. Psychol Bull. 1992;111(3):455.

19. Rosenstock IM, Strecher VJ, Becker MH. Social learning theory and the health belief model. Health Educ Q. 1988;15(2):175-83.

20. Tenkorang EY, Maticka-Tyndale E. Individual-and School-Level Correlates of HIV Testing among Secondary School Students in Kenya. Stud Fam Plan. 2013;44(2):169-87.

21. Prata N, Morris L, Mazive E, Vahidnia F, Stehr M. Relationship between HIV risk perception and condom use: evidence from a population-based survey in Mozambique. Int Fam Plan. Perspect. 2006:192-200.

22. Koh KC, Yong LS. HIV risk perception, sexual behavior, and HIV prevalence among men-who-have-sex-with-men at a communitybased voluntary counseling and testing center in Kuala Lumpur, Malaysia. Interdiscip Perspect Infect Dis. 2014;2014.

23. Tenkorang EY. Perceived vulnerability and HIV testing among youth in cape town, South Africa. Health Promot Int. 2014;31(2):2709.

24. Barden-O'Fallon JL, Bisika T, Sulzbach S, Benson A, Tsui AO. Factors associated with HIV/AIDS knowledge and risk perception in rural Malawi. AIDS Behav. 2004;8(2):131-40.

25. Lindan C, Allen S, Carael M, Nsengumuremyi F, de Perre Van P, Serufilira A, et al. Knowledge, attitudes, and perceived risk of AIDS among urban Rwandan women: relationship to HIV infection and behavior change. AIDS. 1991;5(8):993-1002.

26. Janssen E, van Osch L, de Vries H, Lechner L. Measuring risk perceptions of skin cancer: Reliability and validity of different operationalizations. Br J Health Psychol. 2011;16(1):92-112.

27. Loewenstein GF, Weber EU, Hsee CK, Welch N. Risk as feelings. Psychol Bull. 2001;127(2):267.

28. Armoon B, Noroozi M, Jorjoran Shushtari Z, Sharhani A, Ahounbar E, Karimi S, et al. Factors associated with HIV risk perception among people who inject drugs: Findings from a cross-sectional behavioral survey in Kermanshah, Iran. J Subst Use. 2018;23(1):63-6.

29. Noroozi M, Ahounbar E, eddin Karimi S, Ahmadi S, Najafi M, Bazrafshan A, et al. HIV risk perception and risky behavior among people who inject drugs in Kermanshah, Western Iran. Int J Behav Med. 2017;24(4):613-8.

30. Miller M, Korves C, Fernandez T. The social epidemiology of HIV transmission among African American women who use drugs and their social network members. AIDS Care. 2007;19(7):858-65.

31. Needle RH, Coyle SL, Genser SG, Trotter R. Social networks, drug abuse, and HIV transmission. NIDA Res Monogr. 1995;151.

32. Dowling-Guyer S, Johnson ME, Fisher DG, Needle R, Watters J, Andersen M, et al. Reliability of drug users' self-reported HIV risk behaviors and validity of self-reported recent drug use. Assessment 1994;1(4):383-92.

33. Needle R, Fisher DG, Weatherby N, Chitwood D, Brown B, Cesari $\mathrm{H}$, et al. Reliability of self-reported HIV risk behaviors of drug users. Psychol Addict Behav. 1995;9(4):242.

34. Shokoohi M, Karamouzian M, Mirzazadeh A, Haghdoost A, Rafierad AA, Sedaghat A, et al. HIV knowledge, attitudes, and practices of young people in Iran: findings of a national populationbased survey in 2013. PloS One. 2016;11(9):e0161849.

35. Clifton S, Nardone A, Field N, Mercer CH, Tanton C, Macdowall $\mathrm{W}$, et al. HIV testing, risk perception, and behaviour in the British population. AIDS. 2016;30(6):943

36. Sarker M, Milkowski A, Slanger T, Gondos A, Sanou A, Kouyate $\mathrm{B}$, et al. The role of HIV-related knowledge and ethnicity in determining HIV risk perception and willingness to undergo HIV testing among rural women in Burkina Faso. AIDS Behav. 2005;9(2):243-9.

37. Anderson KG, Beutel AM, Maughan-Brown B. HIV risk perceptions and first sexual intercourse among youth in Cape Town, South Africa. Int Fam Plan Perspect. 2007:98-105.

38. Firth D. Bias reduction of maximum likelihood estimates. Biometrika. 1993;80(1):27-38.

39. Heinze G, Schemper M. A solution to the problem of separation in 
logistic regression. Stat Med. 2002;21(16):2409-19.

40. Greenland S, Mansournia MA, Altman DG. Sparse data bias: a problem hiding in plain sight. BMJ. 2016;352:i1981.

41. Heinze G, Ploner, M. Dunkler, D., \& Southworth, H. Firth's BiasReduced Logistic Regression. R Package, 2016.

42. UNAIDS. Islamic Republic of Iran: 2016, access in http://www.unaids.org/en/regionscountries/countries/islamicrepublico firan [Internet]. 2016 [cited 26 March 2018].

43. Dowson L, Kober C, Perry N, Fisher M, Richardson D. Why some MSM present late for HIV testing: a qualitative analysis. AIDS Care. 2012;24(2):204-9.

44. London AS, Robles A. The co-occurrence of correct and incorrect HIV transmission knowledge and perceived risk for HIV among women of childbearing age in El Salvador. Soc Sci Med. 2000;51(8):1267-78.

45. Napper LE, Fisher DG, Reynolds GL. Development of the Perceived Risk of HIV Scale. AIDS Behav. 2012;16:1075-83.

46. Shokoohi M, Karamouzian M, Khajekazemi R, Osooli M, Sharifi H, Haghdoost AA, et al. Correlates of HIV testing among female sex workers in Iran: Findings of a national bio-behavioural surveillance survey. PloS One. 2016;11(1):e0147587.

47. Rice B, Delpech V, Sadler K, Yin Z, Elford J. HIV testing in black Africans living in England. Epidemiol Infect. 2013;141(8):1741-8.

48. Wingood GM, DiClemente RJ. Application of the theory of gender and power to examine HIV-related exposures, risk factors, and effective interventions for women. Health Educ Behav. 2000;27(5):539-65.

49. Pulerwitz J, Amaro H, Jong WD, Gortmaker SL, Rudd R. Relationship power, condom use and HIV risk among women in the USA. AIDS Care. 2002;14(6):789-800. 